\title{
A demonstration apparatus for the Cartesian diver
}

\author{
J. Güémez ${ }^{\mathrm{a}, 1}$, C. Fiolhais ${ }^{\mathrm{b}, 2}$, and M. Fiolhais ${ }^{\mathrm{b}, 3}$ \\ ${ }^{a}$ Departamento de Física Aplicada, Universidad de Cantabria \\ E-39005 Santander, Spain \\ ${ }^{\mathrm{b}}$ Departamento de Física and Centro de Física Computacional, Universidade de Coimbra \\ P-3004-516 Coimbra, Portugal
}

The Cartesian diver is a nice toy and an intriguing Physics instrument. Recently we have reported an experimental study on the Cartesian diver statics and dynamics [1], using a specially designed apparatus which is much larger than the usual models. The Cartesian diver is an interesting example of the so-called "fold catastrophe", the pressure being the control parameter [1], and this behavior is well observed in our apparatus.

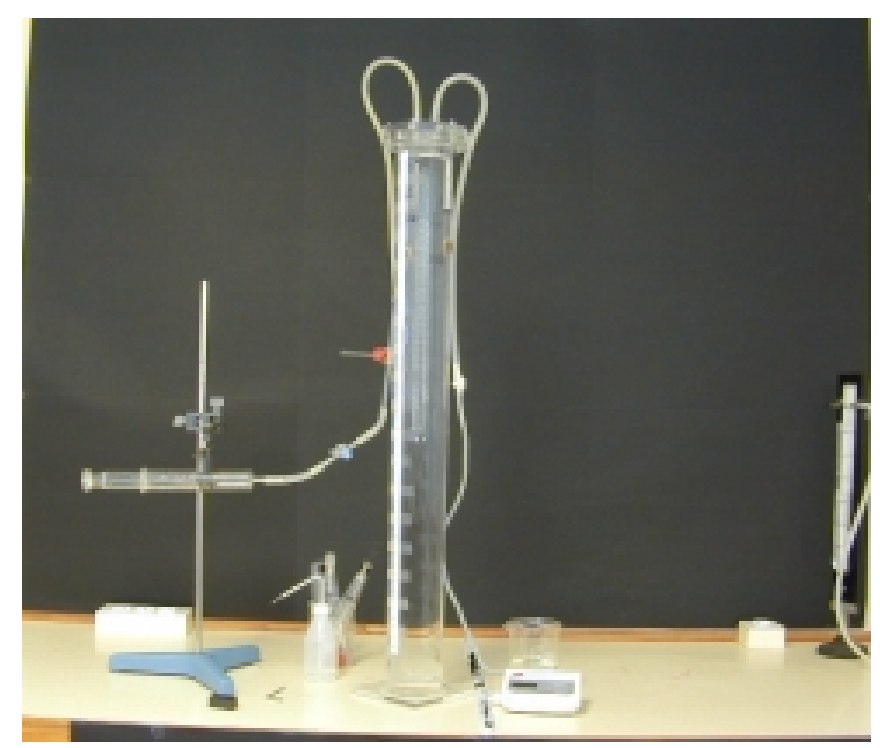

Figure 1 - The Cartesian diver is an inverted test tube with an air bubble trapped inside. The tube is placed in a closed $1 \mathrm{~m}$ long acrylic cylindrical recipient containing water. The pressure on the liquid surface in the recipient is controlled with a syringe (on the left) and measured with a manometer (on the right).

\footnotetext{
1 guemezj@unican.es

2 tcarlos@teor.fis.uc.pt

3 tmanuel@teor.fis.uc.pt
} 
Our Cartesian diver apparatus is made of the following parts (Fig. 1):

(i) A transparent cylindrical tube $1 \mathrm{~m}$ high and $10 \mathrm{~cm}$ diameter almost filled with water. Its upper part was reinforced with a circular crown where a lid is to be fixed with six screws. On the external surface of the cylinder we glued a ruler made from a narrow piece of paper, with a scale;

(ii) A lid with two narrow hollow tubes (Fig. 2). One tube is connected to a syringe, to a oneway stopcock (to control and keep the pressure) and to a three-way stopcock (to control the air admittance), and the other is connected to a digital manometer (a two branch mercury manometer is also appropriate, but to prevent hazards, a medical digital manometer is recommended). The lip has six screw holes symmetrically located and, in its bottom surface, a circular narrow channel, $2 \mathrm{~mm}$ deep and $4 \mathrm{~mm}$ broad, to fit an O-ring;

(iii) Six screws and an O-ring, with diameter $4 \mathrm{~mm}$, to tightly fast the lip to the upper part of the tube, ensuring very good closure;

(iv) A set of different test tubes (the divers) with two metallic clips attached to them (using Scotch tape if the tubes are thick). See Table 1 of [1] for the dimensions of the tubes.

(v) A magnet.

A test tube is partially filled with water closed with a bung and inverted into the water that almost fills up the open cylinder. The bung is then removed and the test tube, with an air bubble trapped inside, floats at the liquid surface. For a typical demonstration with our apparatus, 5 or 6 test tubes, prepared with air bubbles of different sizes, may be accommodated simultaneously. The water level in the cylinder is then adjusted to a reference (zero) level using a suction plastic bottle. Next, the lip with the O-ring is mounted on the mouth of the cylinder and tightly attached to it with the screws (Fig. 2).

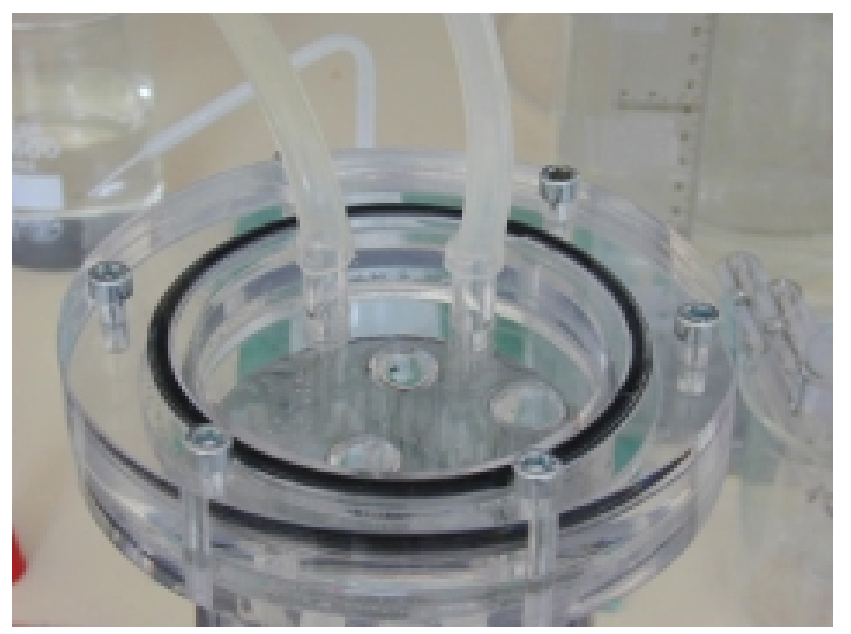

Figure 2 - The top of the recipient, with a removable lip tightly fixed with six screws and an O-ring to ensure good closure. 
The three-way stopcock allows us to control the air volume in the syringe. After this control, its tap should be closed to keep the system isolated from the outside. The syringe allows for a control the pressure on the liquid surface, which may be measured with the manometer. When this pressure is different from the atmospheric pressure, the one way stopcock should be closed to prevent the syringe piston to move.

Archimedes's principle, Pascal's principle, Boyle's law and Newton's law are topics that can be addressed in quantitative demonstrations carried out with the apparatus. We list some studies which may be performed [1]:

- The part of the air bubble below the zero level of the water in the cylindrical recipient is of approximately of the same size for all floating divers, provided we use similar test tubes (Archimedes's principle). The larger the differences in length of the immersed parts of the similar tubes, the larger the difference in size of the trapped bubbles will be.

- A floating diver sinks by increasing the pressure (reducing the volume of air in the syringe) and a sunken diver rises up by reducing the pressure (increasing the volume of air in the syringe). This demonstrates Pascal's principle and Boyle's law. The pressure at which a certain floating diver sinks is slightly different from the pressure at which the sunken diver starts to raise ("constraint catastrophe" [1]).

- The diver, initially in static equilibrium at pressure $P_{0}$, sinks if the pressure increases by $\Delta P$. At atmospheric pressure $P_{0}$, there is a position at distance $x_{\mathrm{nr}}=\Delta P /(\rho g)$ $\left(\rho=1.0 \mathrm{~g} / \mathrm{cm}^{3}\right.$ is the water density and $\left.g=9.8 \mathrm{~m} / \mathrm{s}^{2}\right)$ from the water surface, below which the diver does not return to the surface. If the diver oscillates around the stable equilibrium position and reaches this no return point, it sinks and remains at the bottom. A tall cylindrical tube (1-1.5 $\mathrm{m}$ high) is required for a good observation of this behavior at relatively low pressures.

- The no return point is an unstable equilibrium point, in contrast with the stable equilibrium at the surface (Newton's laws). At fixed pressure, one may find experimentally the no return point (for a given diver, bubble size and pressure), noting that the tube does not move when located exactly at this point, but it comes to the surface or sinks when located above or below that point, respectively. We attached two metal clips symmetrically to the test tube (with scotch-tape). With the aid of a strong magnet operated from the outside the test tube may be moved up or down. In this way the no return point can be experimentally found.

- It is interesting to make a real-time comparison of the numerical and experimental results. The numerical integration of the equations of motion, which are given in [1], requires some parameters, which have to be previously measured. It is important to take 
into account viscous drag effects. We observed that it is enough to consider a force

proportional to the velocity to have good agreement with the experimental results. For the numerical integration we used software with a fast graphical interface (MS Excel).

In conclusion, the described Cartesian diver apparatus may be used to perform quantitative experiments in high schools or in undergraduate laboratory. Both the statics and the dynamics of the system present some interesting and not so well-known aspects.

This work was partially supported by the Ministerio de Ciencia y Tecnología, Spain (Grant BFM2000-1150).

\section{Reference}

[1] J. Güémez, C. Fiolhais, and M. Fiolhais, "The Cartesian Diver and the fold catastrophe”, Am. J. Phys. 70, 710-714 (2002) 